\title{
Research Article \\ Breather Positons and Rogue Waves for the Nonlocal Fokas-Lenells Equation
}

\author{
Chun Wang, Rong Fan, Zhao Zhang, and Biao Li \\ School of Mathematics and Statistics, Ningbo University, Ningbo 315211, China \\ Correspondence should be addressed to Biao Li; libiao@nbu.edu.cn
}

Received 7 March 2021; Accepted 22 April 2021; Published 30 April 2021

Academic Editor: Wen-Xiu Ma

Copyright (C) 2021 Chun Wang et al. This is an open access article distributed under the Creative Commons Attribution License, which permits unrestricted use, distribution, and reproduction in any medium, provided the original work is properly cited.

\begin{abstract}
In this paper, we investigate breather positons and higher-order rogue waves for the nonlocal Fokas-Lenells equation. In this nonlocal optical system, rogue waves can be generated when periods of breather positons go to infinity. In addition, we find two very interesting phenomena: one is that rogue waves sitting on a periodic line wave background are derived; the other is that a hybrid of rogue waves and a periodic kink wave is also constructed. We believe that these interesting findings exist in the optical system corresponding to the nonlocal Fokas-Lenells equation.
\end{abstract}

\section{Introduction}

In the field of mathematics and physics, the research on the degenerate solutions of integrable equations has always been a hot topic. These degenerate solutions include lump solutions, smooth positon solutions, breather positon solutions, and rogue waves. In general, rogue waves localized in both time and space may produce three to five times more amplitude than background waves in a very short time [1]. Rogue waves have also been observed in space plasmas, as well as in optics, when propagating high-power optical radiation through photonic crystal fibers [1-4]. In recent years, many scholars have paid much attention to rogue wave [1] solutions and positon solutions of integrable nonlinear partial differential equations. In 2013, He et al. proposed the generation mechanism of higher-order rogue waves for the nonlinear Schrödinger equation (2). Similarly, their team applied this mechanism to other integrable equations, such as the modified KdV equation (5) and Fokas-Lenells (FL) equation (7), to obtain higher-order rogue waves. Since Matveev [5, 6] proposed singular positon solutions for the Korteweg-de Vries equation in 1992, many experts [7-11] have been working in this field. Among the numerous studies on positons, Wang et al. [10] elaborate the connection between breather positons and rogue waves for a local integrable system.
In this research, we study the nonlocal FL equation [12]:

$$
q_{x t}+q-2 i q q(-x,-t) q_{x}=0
$$

to find some new results. Equation (1) describes the promulgation of ultrashort pulsation in visual fiber. It must be mentioned here that the famous FL equation was proposed by Fokas [13]. Soliton solutions and breather solutions to equation (1) have been given in Ref. [12]. And we have used this nonlocal optical system to obtain some relatively satisfactory results $[14,15]$ : two types of smooth positons with a periodic line wave background and smooth positons with a nonzero background. However, as far as we know, the breather solutions and rogue wave solutions of this important mathematical and physical model (1) have not been studied.

Combining Refs. $[4,12]$, a new solution $q^{[n]}$ of the nonlocal FL equation (1) through $n$-fold Darboux transformation with a plane wave solution $q^{[0]}=a e^{i \theta}, \theta=b x+\left(2 a^{2} b+1\right) t / b$, is given by

$$
q^{[n]}=q^{[0]}-\frac{N_{2 n}}{D_{2 n}},
$$


with

$$
D_{2 n}=\left|\begin{array}{ccccc}
\lambda_{1}^{n} \phi_{1} & \lambda_{1}^{n-1} \varphi_{1} & \cdots & \lambda_{1}^{-(n-2)} \phi_{1} & \lambda_{1}^{-(n-1)} \varphi_{1} \\
\lambda_{2}^{n} \phi_{2} & \lambda_{2}^{n-1} \varphi_{2} & \cdots & \lambda_{2}^{-(n-2)} \phi_{2} & \lambda_{2}^{-(n-1)} \varphi_{2} \\
\vdots & \vdots & \ddots & \vdots & \vdots \\
\lambda_{2 n-1}^{n} \phi_{2 n-1} & \lambda_{2 n-1}^{n-1} \varphi_{2 n-1} & \cdots & \lambda_{2 n-1}^{-(n-2)} \phi_{2 n-1} & \lambda_{2 n-1}^{-(n-1)} \varphi_{2 n-1} \\
\lambda_{2 n}^{n} \phi_{2 n} & \lambda_{2 n}^{n-1} \varphi_{2 n} & \cdots & \lambda_{2 n}^{-(n-2)} \phi_{2 n} & \lambda_{2 n}^{-(n-1)} \varphi_{2 n}
\end{array}\right|,
$$

where

$$
\begin{aligned}
\phi_{j} & =\left(2 a b \lambda_{j} e^{i \sigma \Omega}+\left(-\lambda_{j}^{2}+b+2 \sigma\right) e^{-i \sigma \Omega}\right) e^{i \theta / 2} \\
\varphi_{j} & =\left(\left(-\lambda_{j}^{2}+b+2 \sigma\right) e^{i \sigma \Omega}+2 a b \lambda_{j} e^{-i \sigma \Omega}\right) e^{-i \theta / 2} \\
\Omega & =-\frac{t}{b \lambda_{j}^{2}}+x \\
\sigma & =\frac{1}{2} \sqrt{\lambda_{j}^{4}+\left(-4 a^{2} b^{2}-2 b\right) \lambda_{j}^{2}+b^{2}}
\end{aligned}
$$

Here, $a \in \mathbb{C}$ and $b \in \mathbb{R}$. And $N_{2 n}$ is a determinant given by $D_{2 n}$ through replacing its last column with vector $\left[\begin{array}{lllll}\lambda_{1}^{-n} \phi_{1} & \lambda_{2}^{-n} \phi_{2} & \cdots & \lambda_{2 n-1}^{-n} \phi_{2 n-1} & \lambda_{2 n}^{-n} \phi_{2 n}\end{array}\right]^{T}$.

In the second section, we will show that the relation between breather positons and rogue waves mentioned in Ref. [10] still holds true for nonlocal integrable systems. Then, we apply different constraints and degenerate mechanisms to equation (2) to produce some novel exact solutions, like rogue waves sitting on a periodic line wave background, a nonlinear superposition between rogue waves and a periodic kink wave.

\section{The Relation between Breather Positons and Rogue Waves}

Although in Refs. [10, 16] the authors obtained breather positons in the local integrable system, these ideas are still valid in the nonlocal system studied in this paper. Simply put, the breather positon is a limit case of equation (2). Hence, we give the following proposition about breather positons without proof. At the same time, we may wish to set $\{a=\alpha i, b=1, \alpha \in \mathbb{R}\}$ in order to facilitate discussion and simplify the calculation.

Proposition 1. Based on the degenerate Darboux transformation, the nth-order breather positons $q_{n-b p}$ are given by

$$
q_{n-b p}=q^{[0]}-\frac{N_{2 n}{ }^{\prime}}{D_{2 n}{ }^{\prime}},
$$

with

$$
\begin{gathered}
N_{2 n}{ }^{\prime}=\left(\left.\frac{\partial^{h(i)}}{\partial \varepsilon^{h(i)}}\right|_{\varepsilon=0}\left(N_{2 n}\right)_{i j}\left(\lambda_{j}+\varepsilon^{2}\right)\right)_{2 n \times 2 n}, \\
D_{2 n}{ }^{\prime}=\left(\left.\frac{\partial^{h(i)}}{\partial \varepsilon^{h(i)}}\right|_{\varepsilon=0}\left(D_{2 n}\right)_{i j}\left(\lambda_{j}+\varepsilon^{2}\right)\right)_{2 n \times 2 n}, \\
h(x)=2\left[\frac{x-1}{2}\right],
\end{gathered}
$$

where

$$
\begin{gathered}
\alpha \in[-1,1], \\
\lambda_{2 j-1}=-\lambda_{2 j}^{*}=\lambda_{1}, \quad j=1, \cdots, n .
\end{gathered}
$$

Here, $[x]$ denotes the floor function of $x$, and $D_{2 n}, N_{2 n}$, and $q^{[0]}$ are given by equation (2).

From Figure 1, we can have a general understanding of the spatiotemporal characteristics of breather positons. Since breather positons were proposed by Wang et al. [10], it has been a difficult problem to study the change trend of breather positons over time. It is always a difficult problem to find the trajectories of breather positons $[10,16,17]$. Not to mention, let us study the dynamic properties of breather positons like decomposing smooth positons [7-9].

When $\lambda=b a \pm \sqrt{a^{2} b^{2}+b}$, we find $\phi_{j}=0$ and $\varphi_{j}=0$. This implies that eigenfunctions $\left\{\phi_{j}, \varphi_{j}\right\}$ degenerate at $\lambda=b a \pm$ $\sqrt{a^{2} b^{2}+b}$. In particular, when $\{b=1, a=\alpha i, \alpha \in[-1,1]\}$, we can get $b a+\sqrt{a^{2} b^{2}+b}=-\left(b a-\sqrt{a^{2} b^{2}+b}\right)^{*}$. Combining with the main idea of Ref. [10], we can say that the period of breather positons described by Proposition 1 goes to infinity when $\left\{\lambda_{1} \rightarrow b a+\sqrt{a^{2} b^{2}+b} a=\alpha i, \alpha \in[-1,1]\right\}$. In this particular case just mentioned, breather positons are converted to rogue waves.

Proposition 2. Based on the degenerate Darboux transformation, the nth-order rogue waves $q_{n-R W}$ are given by

$$
q_{n-R W}=q^{[0]}-\frac{N_{2 n}{ }^{\prime}}{D_{2 n}{ }^{\prime}},
$$

with

$$
\begin{gathered}
N_{2 n}{ }^{\prime}=\left(\left.\frac{\partial^{h(i)}}{\partial \varepsilon^{h(i)}}\right|_{\varepsilon=0}\left(N_{2 n}\right)_{i j}\left(\lambda_{j}+\varepsilon^{2}\right)\right)_{2 n \times 2 n}, \\
D_{2 n}{ }^{\prime}=\left(\left.\frac{\partial^{h(i)}}{\partial \varepsilon^{h(i)}}\right|_{\varepsilon=0}\left(D_{2 n}\right)_{i j}\left(\lambda_{j}+\varepsilon^{2}\right)\right)_{2 n \times 2 n}, \\
h(x)=2\left[\frac{x+1}{2}\right] .
\end{gathered}
$$




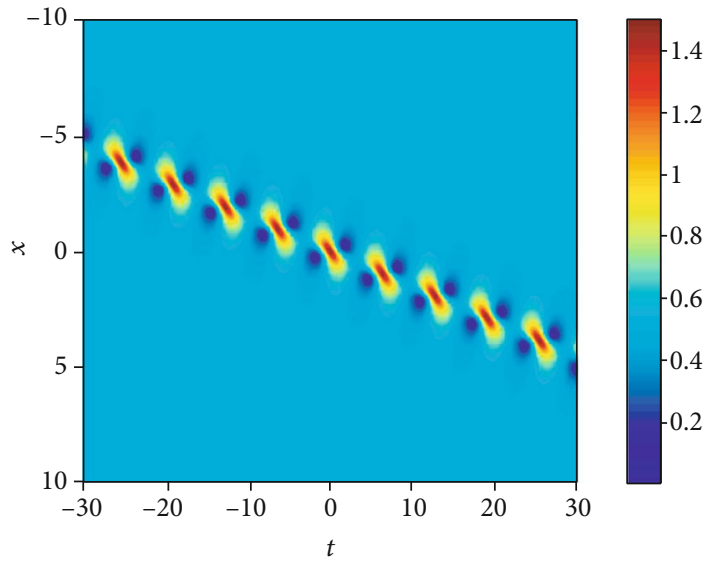

(a)

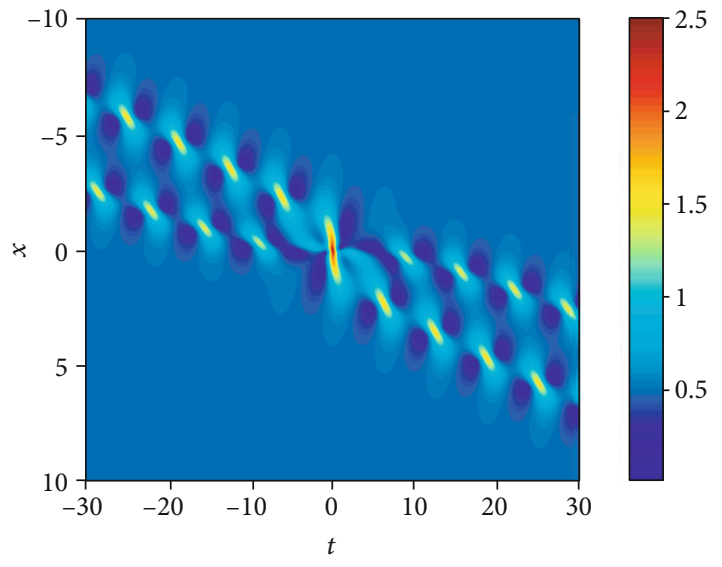

(b)

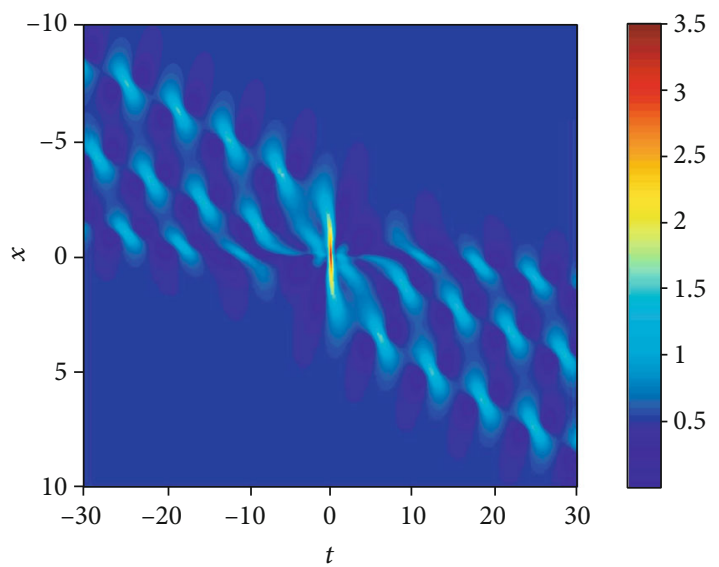

(c)

Figure 1: Breather positons described by Proposition 1 with parameter selections $a=i / 2, b=1$, and $\lambda_{1}=1+i$ (color online). (a) A first-order breather positon $\left|q_{1-\mathrm{bp}}\right|$. (b) A second-order breather positon $\left|q_{2-\mathrm{bp}}\right|$. (c) A third-order breather positon $\left|q_{3-\mathrm{bp}}\right|$.

where

$$
\begin{gathered}
\alpha \in[-1,1], \\
\lambda_{2 j-1}=-\lambda_{2 j}^{*}=\lambda_{1}=b a+\sqrt{a^{2} b^{2}+b}, \quad j=1, \cdots, n .
\end{gathered}
$$

Remark 3. There are many conclusions $[2,3,7,10,11,16]$ like Propositions 1 and 2, so this research will not prove them in detail. Essentially, all the propositions in this paper are different limit cases of equation (2). The most difficult step to derive these propositions is to obtain the constraints such as equation (10) and the function $h(x)$ that controls the degree of degradation.

Figures 1 and 2 intuitively illustrate that the transformation relationship between breather positons and rogue waves mentioned in Ref. [10] is still valid in the nonlocal FL system. As $\lambda_{1}$ approaches to $b a+\sqrt{a^{2} b^{2}+b}$, the other peaks near the center region of breather positons gradually disappear until only the central profile survives. In other words, as the period of a breather positon goes to infinity, its central region will turn into a rogue wave.

According to Proposition 2, the mathematical expression of a first-order rogue wave $q_{1-\mathrm{RW}}$ can be derived:

$q_{1-\mathrm{RW}}=\frac{-i\left(16 \alpha^{4} t x+4 i \alpha^{2} t+12 i \alpha^{2} x+4 \alpha^{2} t^{2}-8 \alpha^{2} t x+4 \alpha^{2} x^{2}-3\right) \alpha e^{-i\left(2 \alpha^{2} t-t-x\right)}}{16 \alpha^{4} t x+4 i \alpha^{2} t-4 i \alpha^{2} x+4 \alpha^{2} t^{2}-8 \alpha^{2} t x+4 \alpha^{2} x^{2}+1}$.

$\left|q_{1-\mathrm{RW}}\right|$ has three critical points. $\left|q_{1-\mathrm{RW}}\right|$ reaches the maximum value $3|\alpha|$ at point $\{x=0, t=0\}$; the minimum value 0 is obtained at points $\left\{t=-3 \sqrt{3} /\left(4 \sqrt{-3 \alpha^{2}+4} \alpha\right)\right.$, $\left.x=\sqrt{3} /\left(4 \sqrt{-3 \alpha^{2}+4} \alpha\right)\right\}$ and $\left\{t=3 \sqrt{3} /\left(4 \sqrt{-3 \alpha^{2}+4} \alpha\right), x\right.$ $\left.=-\sqrt{3} /\left(4 \sqrt{-3 \alpha^{2}+4} \alpha\right)\right\}$. When $\{x \rightarrow \infty, t \rightarrow \infty\}$, it is easy to find that $\left|q_{1-\mathrm{RW}}\right|=|\alpha|$.

When $n=2$, a second-order rogue wave $q_{2-\mathrm{RW}}$ can be derived from Proposition 2:

$$
q_{2-\mathrm{RW}}=\left(\alpha i-\frac{L_{1}}{L_{2}}\right) e^{-i\left(2 \alpha^{2} t-t-x\right)},
$$




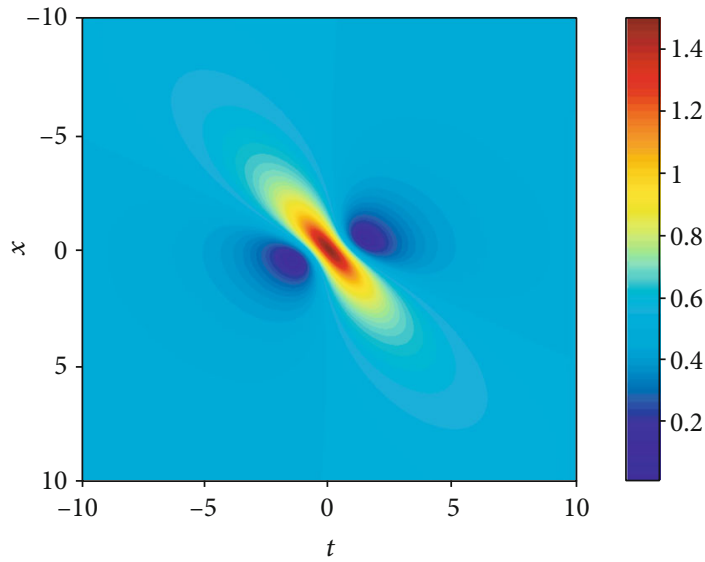

(a)

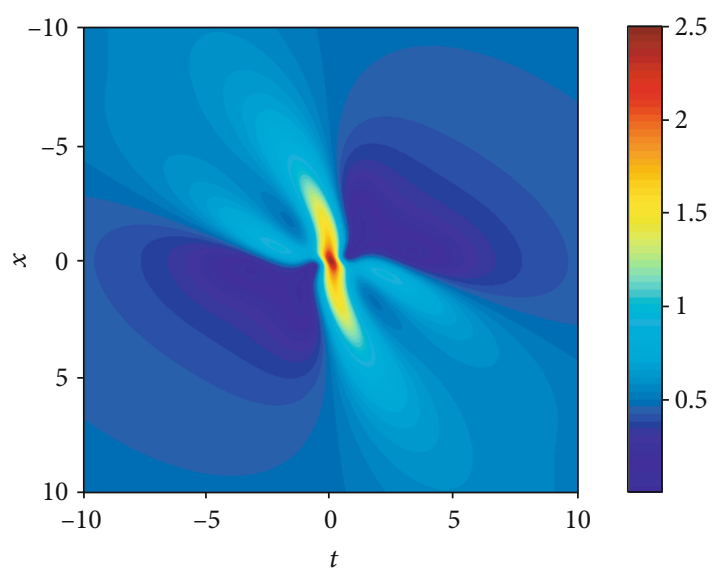

(b)

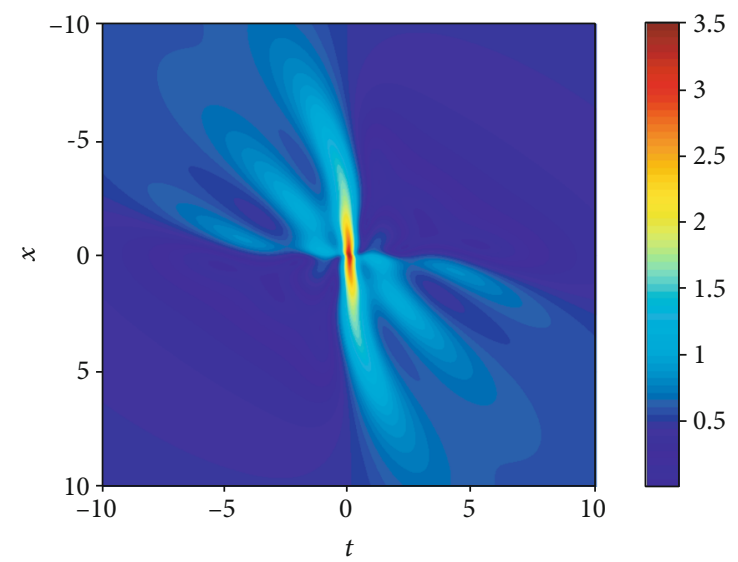

(c)

Figure 2: Rogue waves described by Proposition 2 with parameters $\lambda_{1}=(i / 2)+(\sqrt{3} / 2), a=i / 2$, and $b=1$ (color online). (a) First-order rogue wave. (b) Second-order rogue wave. (c) Third-order rogue wave.

where

$$
\begin{aligned}
L_{1}= & 768 \alpha^{7} x^{5}+6144 \alpha^{9} x^{4} t-3072 \alpha^{7} x^{4} t-12288 \alpha^{9} x^{3} t^{2} \\
& +12288 \alpha^{11} x^{3} t^{2}+4608 \alpha^{7} x^{3} t^{2}+6144 \alpha^{9} x^{2} t^{3} \\
& -3072 \alpha^{7} x^{2} t^{3}+768 \alpha^{7} x t^{4}+1536 i \alpha^{7} x^{4}+192 i \alpha^{5} x^{4} \\
& -768 i \alpha^{5} t x^{3}+6144 i \alpha^{9} x^{3} t+1152 i \alpha^{5} x^{2} t^{2} \\
& +9216 i \alpha^{9} x^{2} t^{2}-4608 i \alpha^{7} t^{2} x^{2}-768 i \alpha^{5} t^{3} x \\
& +3072 i \alpha^{7} x t^{3}+192 i \alpha^{5} t^{4}-768 \alpha^{7} x^{3}-768 \alpha^{5} x^{3} \\
& -4608 \alpha^{7} x^{2} t+1152 \alpha^{5} x^{2} t+6912 \alpha^{7} x t^{2}-384 \alpha^{5} t^{3} \\
& -576 i \alpha^{5} x^{2}+288 i \alpha^{3} x^{2}-576 i \alpha^{3} t x+3456 i \alpha^{5} x t \\
& +576 i \alpha^{5} t^{2}+288 i \alpha^{3} t^{2}-432 \alpha^{3} x-288 \alpha^{3} t-36 i \alpha,
\end{aligned}
$$

$$
\begin{aligned}
L_{2}= & 64 \alpha^{6} x^{6}+768 \alpha^{8} x^{5} t-384 \alpha^{6} x^{5} t+3072 \alpha^{10} x^{4} t^{2} \\
& -3072 \alpha^{8} x^{4} t^{2}+960 \alpha^{6} x^{4} t^{2}-6144 \alpha^{10} x^{3} t^{3} \\
& +4096 \alpha^{12} x^{3} t^{3}+4608 \alpha^{8} x^{3} t^{3}-1280 \alpha^{6} x^{3} t^{3} \\
& +3072 \alpha^{10} x^{2} t^{4}-3072 \alpha^{8} x^{2} t^{4}+960 \alpha^{6} x^{2} t^{4}+768 \alpha^{8} x t^{5} \\
& -384 \alpha^{6} x t^{5}+64 \alpha^{6} t^{6}-192 i \alpha^{6} x^{5}+960 i \alpha^{6} x^{4} t \\
& -1536 i \alpha^{8} t x^{4}-1920 i \alpha^{6} t^{2} x^{3}-3072 i \alpha^{10} t^{2} x^{3}
\end{aligned}
$$

$$
\begin{aligned}
& +4608 i \alpha^{8} x^{3} t^{2}+1920 i \alpha^{6} x^{2} t^{3}+3072 i \alpha^{10} x^{2} t^{3} \\
& -4608 i \alpha^{8} t^{3} x^{2}-960 i \alpha^{6} t^{4} x+1536 i \alpha^{8} x t^{4}+192 i \alpha^{6} t^{5} \\
& +48 \alpha^{4} x^{4}-192 \alpha^{6} x^{4}-192 \alpha^{4} x^{3} t-384 \alpha^{6} x^{3} t \\
& +2304 \alpha^{8} x^{3} t+288 \alpha^{4} x^{2} t^{2}+1152 \alpha^{6} x^{2} t^{2}+2304 \alpha^{8} x^{2} t^{2} \\
& -192 \alpha^{4} x t^{3}-384 \alpha^{6} x t^{3}+2304 \alpha^{8} x t^{3}+48 \alpha^{4} t^{4} \\
& -192 \alpha^{6} t^{4}-192 i \alpha^{6} x^{3}-96 i \alpha^{4} x^{3}+288 i \alpha^{4} x^{2} t \\
& +1728 i \alpha^{6} x^{2} t-288 i \alpha^{4} t^{2} x-1728 i \alpha^{6} t^{2} x+192 i \alpha^{6} t^{3} \\
& +96 i \alpha^{4} t^{3}+144 \alpha^{4} x^{2}+108 \alpha^{2} x^{2}-216 \alpha^{2} x t+1296 \alpha^{4} x t \\
& +144 \alpha^{4} t^{2}+108 \alpha^{2} t^{2}-108 i \alpha^{2} x+108 i \alpha^{2} t+9 .
\end{aligned}
$$

The maximum amplitude of $\left|q_{2-\mathrm{RW}}\right|$ is $5|\alpha|$ at $(0,0)$. In addition to the maximum value, $\left|q_{2-\mathrm{RW}}\right|$ has four other local maximum points, which can be easily found in Figure 2(b).

The mathematical expressions for higher-order rogue waves are too verbose to be given in this paper. By setting $\{t=0, x=0\}$, the maximum amplitude of $\left|q_{n-\mathrm{RW}}\right|$ is $(2 n$ $+1)|\alpha|$. On each side of the straight line $t=0,\left|q_{n-\mathrm{RW}}\right|$ has $(n(n+1) / 2)-1$ local maximum points. The following 


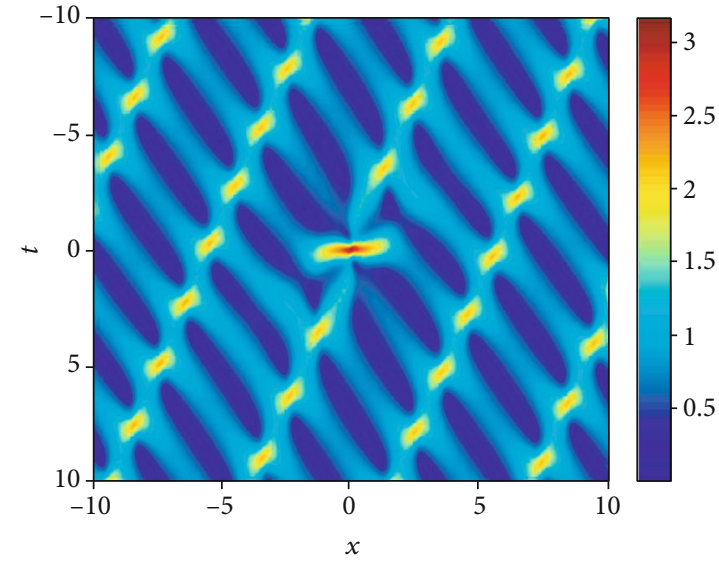

(a)

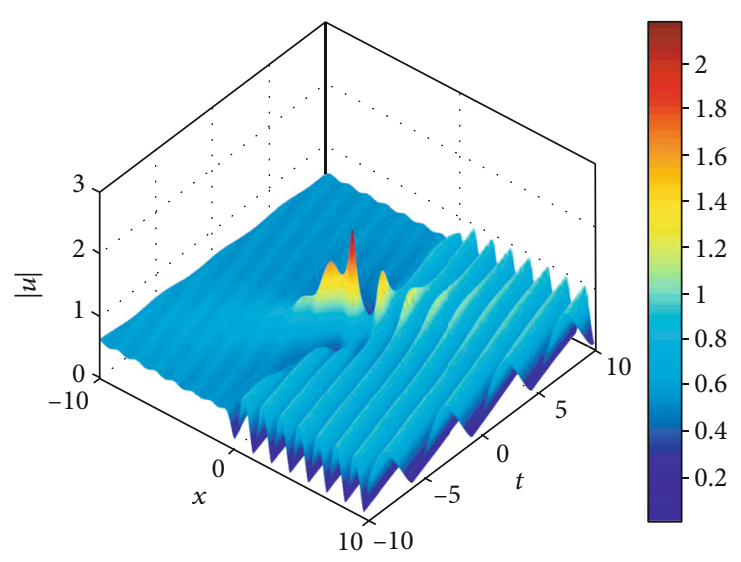

(b)

Figure 3: (a) A first-order rogue wave sitting on periodic line waves described by Proposition 4 with parameters $n=1, a=i / 2, \lambda_{1}=i, \lambda_{2}=3 i / 2$, and $\lambda_{3}=3 / 2+\sqrt{3} / 2$. (b) A nonlinear superposition between a first-order rogue wave and a periodic kink wave described by Proposition 4 with parameter selections $n=1, a=i / 2, \lambda_{1}=1-3 i, \lambda_{2}=3 i$, and $\lambda_{3}=3 / 2+\sqrt{3} / 2$. Color online.

conclusion about higher-order rogue waves can also be found in Figure 2. Starting from $|t|=\infty$, before the maximum amplitude in the center, there are a series of peaks in $\left|q_{n-\mathrm{RW}}\right|$ and the height of these peaks gradually increases. Comparing the relevant conclusions in Ref. [3], the basic description of the rogue waves in the nonlocal FL system is roughly the same as that in the local system.

To be honest, Propositions 1 and 2 simply apply the mature method $[3,4,10]$ to the new equation. The rogue waves shown in Figure 2 are technically known as fundamental patterns of rogue waves. However, the nonlocal equation studied in this paper must satisfy the condition $\left\{\phi_{j}(x, t)=\right.$ $\left.\varphi_{j}(-x,-t), j=1, \cdots, 2 n\right\}[12]$. Therefore, a small perturbation parameter cannot be introduced to generate triangular patterns and ring patterns as in Refs. [3, 4, 18].

\section{Rogue Waves Sitting on Other Forms of Waves}

A rogue wave with a plane wave background is commonplace [1-4]. In order to better explain some phenomena in mathematical and physical models, some scholars have explored the generation mechanism of hybrid solutions consisting of rogue waves and breather solutions $[10,18]$.

In equation (2), there is no constraint relation between $\lambda_{2 j}$ and $\lambda_{2 j-1}$. This is the biggest difference between the nonlocal FL systems and the local systems in Refs. [1-4, 18]. Hence, a hybrid of rogue waves and other forms of solutions can be derived. We have the following conclusion.

Proposition 4. Based on the degenerate Darboux transformation, a novel nonlinear superposition between the nth-order rogue waves and other forms of solutions $q_{n-R W-h y b}$ is given by

$$
q_{n-R W-h y b}=q^{[0]}-\frac{N_{2 n}{ }^{\prime}}{D_{2 n}{ }^{\prime}},
$$

with

$$
\begin{aligned}
& N_{2 n}{ }^{\prime}=\left(\left.\frac{\partial^{h(i)}}{\partial \varepsilon^{h(i)}}\right|_{\varepsilon=0}\left(N_{2 n}\right)_{i j}\left(\lambda_{j}+\varepsilon^{2}\right)\right)_{2 n \times 2 n}, \\
& D_{2 n}{ }^{\prime}=\left(\left.\frac{\partial^{h(i)}}{\partial \varepsilon^{h(i)}}\right|_{\varepsilon=0}\left(D_{2 n}\right)_{i j}\left(\lambda_{j}+\varepsilon^{2}\right)\right)_{2 n \times 2 n}, \\
& h(x)=\left(\begin{array}{ll}
0, & x \leq 2, \\
2\left[\frac{x+1}{2}\right]-2, & x>2,
\end{array}\right.
\end{aligned}
$$

where

$$
\begin{aligned}
\alpha & \in[-1,1], \\
\lambda_{1} & =\xi_{1}+\eta_{1} i, \\
\lambda_{2} & =\xi_{2}+\eta_{2} i, \\
\lambda_{2 j-1} & =-\lambda_{2 j}^{*}=\lambda_{3}=b a+\sqrt{a^{2} b^{2}+b}, \quad j=2 \cdots n+1 .
\end{aligned}
$$

According to relevant conclusions in Refs. [12, 15], we can get rogue waves sitting on periodic line waves when $\left\{\xi_{1}\right.$ $\left.=0, \xi_{2}=0\right\}$. A nonlinear superposition between rogue waves and a periodic kink wave can be derived when $\left\{\xi_{2}=0, \eta_{1}=\right.$ $\left.-\eta_{2}\right\}$. We have to say that it is difficult to give constraints that guarantee the analyticity of hybrid solutions described by Proposition 4. On the basis of some conclusions [12, 15], the improvement of Proposition 4 is to get rogue waves sitting on periodic line waves or a periodic kink wave. If the precondition $\left\{\xi_{1}=0, \xi_{2}=0\right\}$ or $\left\{\xi_{2}=0, \eta_{1}=-\eta_{2}\right\}$ is satisfied and the solution is an analytic function, then the solution shown in Figure 3 will be derived. As far as we know, the interaction between rogue waves and breathers is relatively 
common [18], but the results shown in Figure 3 have not been found.

\section{Conclusion}

In addition to studying the breather positons and rogue waves for the nonlocal Fokas-Lenells equation, we also find some new and interesting results shown in Figure 3: rogue waves sitting on periodic line waves and a hybrid of rogue waves and a periodic kink wave. Figures 1 and 2 vividly illustrate that a rogue wave can be generated when the period of a breather positon goes to infinity. We have tried our best to analyze the dynamic properties of rogue waves from two aspects: specific mathematical expressions (equations (11) and (12)) and exquisite images (Figure 2).

Although our research is concise and the graphics are beautiful, the dynamic properties of these breather positon solutions are worthy of further study. Can we find the trajectories of breather positons as in Ref. [17]? If this works, then we can make some important conclusions about the dynamics. Can the $n$ th-order breather positons be approximately decomposed into $n$ single breather waves with the phase shift when $|t| \rightarrow \infty$ ? Can we find a constraint that guarantees that the solution represented by Proposition 4 is analytic? Further, how do we investigate the dynamic properties of singular solutions in Proposition 4? As far as we know, this problem has not been resolved. We look forward to many colleagues working together to solve them.

\section{Data Availability}

No data were used to support this study.

\section{Conflicts of Interest}

The authors declare that they have no conflicts of interest.

\section{Acknowledgments}

This work is supported by the National Natural Science Foundation of China under Grant Nos. 11775121 and 11435005 and K.C.Wong Magna Fund in Ningbo University.

\section{References}

[1] A. Chabchoub, N. P. Hoffmann, and N. Akhmediev, "Rogue wave observation in a water wave tank," Physical Review Letters, vol. 106, no. 20, p. 204502, 2011.

[2] J. S. He, H. R. Zhang, L. H. Wang, K. Porsezian, and A. S. Fokas, "Generating mechanism for higher-order rogue waves," Physical Review E, vol. 87, no. 5, 2013.

[3] J. S. He, L. H. Wang, L. J. Li, K. Porsezian, and R. Erdélyi, "Few-cycle optical rogue waves: complex modified Kortewegde Vries equation," Physical Review E, vol. 89, no. 6, 2014.

[4] S. Xu, J. S. He, Y. Cheng, and K. Porseiza, "The n-order rogue waves of Fokas-Lenells equation," Mathematicsl Methods in the Applied Sciences, vol. 38, no. 6, pp. 1106-1126, 2015.

[5] V. B. Matveev, "Generalized Wronskian formula for solutions of the KdV equations: first applications," Physics Letters A, vol. 166, no. 3-4, pp. 205-208, 1992.
[6] V. B. Matveev, "Positon-positon and soliton-positon collisions: KdV case," Physics Letters A, vol. 166, no. 3-4, pp. 209-212, 1992.

[7] W. Liu, Y. S. Zhang, and J. S. He, "Dynamics of the smooth positons of the complex modified KdV equation," Wave Random Complex, vol. 28, no. 2, pp. 203-214, 2018.

[8] W. J. Song, S. W. Xu, M. H. Li, and J. S. He, "Generating mechanism and dynamic of the smooth positons for the derivative nonlinear Schrödinger equation," Nonlinear Dynamics, vol. 97, no. 4, pp. 2135-2145, 2019.

[9] Q. X. Xing, Z. W. Wu, D. Mihalache, and J. S. He, "Smooth positon solutions of the focusing modified Korteweg-de Vries equation," Nonlinear Dynamics, vol. 89, no. 4, pp. 22992310, 2017.

[10] L. H. Wang, J. S. He, H. Xu, J. Wang, and K. Porsezian, “Generation of higher-order rogue waves from multibreathers by double degeneracy in an optical fiber," Physical Review E, vol. 95, no. 4, 2017.

[11] Z. Zhang, X. Y. Yang, and B. Li, "Soliton molecules and novel smooth positons for the complex modified KdV equation," Applied Mathematics Letters, vol. 103, p. 106168, 2020.

[12] Q. Y. Zhang, Y. Zhang, and R. S. Ye, "Exact solutions of nonlocal Fokas-Lenells equation," Applied Mathematics Letters, vol. 98, pp. 336-343, 2019.

[13] A. S. Fokas, "On a class of physically important integrable equations," Physica D, vol. 87, no. 1-4, pp. 145-150, 1995.

[14] R. Fan, Z. Zhang, and B. Li, "Multisoliton solutions with even numbers and its generated solutions for nonlocal FokasLenells equation," Communications in Theoretical Physics, vol. 72, no. 12, p. 125007, 2020.

[15] B. Wang, Z. Zhang, and B. Li, "Two types of smooth positons for nonlocal Fokas-Lenells equation," International Journal of Modern Physics B, vol. 34, no. 17, p. 2050148, 2020.

[16] D. Q. Qiu and W. G. Cheng, "The nth-order degenerate breather solution for the Kundu-Eckhaus equation," Applied Mathematics Letters, vol. 98, pp. 13-21, 2019.

[17] Z. Zhang, X. Y. Yang, and B. Li, "Novel soliton molecules and breather-positon on zero background for the complex modified KdV equation," Nonlinear Dynamics, vol. 100, no. 2, pp. 1551-1557, 2020.

[18] H. A. Lin, J. S. He, L. H. Wang, and D. Mihalache, "Several categories of exact solutions of the third-order flow equation of the Kaup-Newell system," Nonlinear Dynamics, vol. 100, no. 3, pp. 2839-2858, 2020. 